PROCEEDINGS OF THE

AMERICAN MATHEMATICAL SOCIETY

Volume 135, Number 2, February 2007, Pages 469-478

S 0002-9939(06)08498-X

Article electronically published on August 10, 2006

\title{
ON FRAMES FOR COUNTABLY GENERATED HILBERT $C^{*}$-MODULES
}

\author{
LJILJANA ARAMBAŠIĆ
}

(Communicated by Joseph A. Ball)

\begin{abstract}
Let $V$ be a countably generated Hilbert $C^{*}$-module over a $C^{*}$ algebra $A$. We prove that a sequence $\left\{f_{i}: i \in I\right\} \subseteq V$ is a standard frame for $V$ if and only if the sum $\sum_{i \in I}\left\langle x, f_{i}\right\rangle\left\langle f_{i}, x\right\rangle$ converges in norm for every $x \in V$ and if there are constants $C, D>0$ such that $C\|x\|^{2} \leq\left\|\sum_{i \in I}\left\langle x, f_{i}\right\rangle\left\langle f_{i}, x\right\rangle\right\| \leq$ $D\|x\|^{2}$ for every $x \in V$. We also prove that surjective adjointable operators preserve standard frames. A class of frames for countably generated Hilbert $C^{*}$-modules over the $C^{*}$-algebra of all compact operators on some Hilbert space is discussed.
\end{abstract}

\section{INTRODUCTION AND PRELIMINARIES}

A (right) Hilbert $C^{*}$-module $V$ over a $C^{*}$-algebra $A$ (or a Hilbert $A$-module) is a linear space which is a right $A$-module, together with an $A$-valued inner product $\langle\cdot, \cdot\rangle$ on $V \times V$ which is linear in the second and conjugate linear in the first variable such that $V$ is a Banach space with the norm $\|x\|=\|\langle x, x\rangle\|^{1 / 2}$. We use the symbol $\langle V, V\rangle$ for the closed, two-sided ideal of $A$ spanned by all inner products $\langle x, y\rangle$, $x, y \in V . V$ is said to be a full Hilbert $A$-module if $\langle V, V\rangle=A$.

We denote the $C^{*}$-algebra of all adjointable operators on a Hilbert $C^{*}$-module $V$ by $\mathbf{B}(V)$. We also use $\mathbf{B}(V, W)$ to denote the space of all adjointable operators acting between different Hilbert $A$-modules. A good reference for Hilbert $C^{*}$-modules are the lecture notes of E. C. Lance [12].

The $C^{*}$-algebra of all bounded operators and the ideal of all compact operators on a Hilbert space $H$ are denoted by $\mathbf{B}(H)$ and $\mathbf{K}(H)$, respectively.

Frames for Hilbert spaces were introduced by Duffin and Schaeffer [6] as part of their research in non-harmonic Fourier series. A frame for a separable Hilbert space $H$ is defined to be a finite or countable sequence $\left\{f_{i}: i \in I\right\}$ for which there exists constants $C, D>0$ such that

$$
C\|x\|^{2} \leq \sum_{i \in I}\left|\left(x, f_{i}\right)\right|^{2} \leq D\|x\|^{2}, \quad x \in H .
$$

M. Frank and D. Larson [7, 8, generalized this definition to the situation of countably generated Hilbert $C^{*}$-modules. A frame for a countably generated Hilbert

Received by the editors July 30, 2005 and, in revised form, September 19, 2005.

2000 Mathematics Subject Classification. Primary 46L99; Secondary 46L05, 46H25.

Key words and phrases. $C^{*}$-algebra, Hilbert $C^{*}$-module, frame, frame transform, frame operator, compact operator.

(C)2006 American Mathematical Society Reverts to public domain 28 years from publication 
$C^{*}$-module $V$ is a sequence $\left\{f_{i}: i \in I\right\}$ ( $I \subseteq \mathbf{N}$ finite or countable) for which there are constants $C, D>0$ such that

$$
C\langle x, x\rangle \leq \sum_{i \in I}\left\langle x, f_{i}\right\rangle\left\langle f_{i}, x\right\rangle \leq D\langle x, x\rangle, \quad x \in V .
$$

We consider standard frames for which the sum in the middle of (1.1) converges in norm for every $x \in V$. (For non-standard frames the sum in (1.1) converges only weakly for at least one element of $V$.) The numbers $C$ and $D$ are called frame bounds. A frame $\left\{f_{i}: i \in I\right\}$ is called a tight frame if we can choose $C=D$ and a Parseval frame (or a normalized tight frame) if $C=D=1$. A sequence which satisfies only the right-hand inequality in (1.1) is called a Bessel sequence with a Bessel bound D.

The frame transform for a standard frame $\left\{f_{i}: i \in I\right\}$ is the map $\theta: V \rightarrow \ell_{2}(A)$ defined by $\theta(x)=\left(\left\langle f_{i}, x\right\rangle\right)_{i}$, where $\ell_{2}(A)$ denotes a Hilbert $A$-module $\left\{\left(a_{i}\right)_{i}: a_{i} \in\right.$ $A, \sum_{i \in I} a_{i}^{*} a_{i}$ converges in norm $\}$ with pointwise operations and the inner product $\left\langle\left(a_{i}\right)_{i},\left(b_{i}\right)_{i}\right\rangle=\sum_{i \in I} a_{i}^{*} b_{i}$. The frame transform possesses an adjoint operator and realizes an embedding of $V$ onto an orthogonal summand of $\ell_{2}(A)$ ( 8 , Theorem 4.4]). The operator $S=\left(\theta^{*} \theta\right)^{-1} \in \mathbf{B}(V)$ is said to be the frame operator for a standard frame $\left\{f_{i}: i \in I\right\}$. The frame operator is positive, invertible, and is the unique operator in $\mathbf{B}(V)$ such that the reconstruction formula

$$
x=\sum_{i \in I} f_{i}\left\langle S f_{i}, x\right\rangle
$$

holds for all $x \in V$. Let us remark that although M. Frank and D. Larson [7, 8] stated all their results for the unital case, many proofs can be applied to the non-unital situation.

In a countably generated Hilbert $C^{*}$-module over a unital $C^{*}$-algebra, standard frames always exist 7 . Also, a Hilbert $C^{*}$-module over a $C^{*}$-algebra of all compact operators $\mathbf{K}(H)$ on some Hilbert space $H$ possesses frames; this follows from [2], where the concept of an orthonormal basis for a Hilbert $C^{*}$-module was discussed.

An element $v$ of a Hilbert $A$-module $V$ is said to be a basic vector if $e=\langle v, v\rangle$ is a projection in $A$ such that $e A e=\mathbf{C} e$. The system of basic vectors $\left\{v_{i}: i \in I\right\}$ in $V$ is said to be an orthonormal basis for $V$ if $\left\langle v_{i}, v_{j}\right\rangle=0$ for all $i \neq j$, and if it generates a dense submodule of $V$. Every orthonormal basis $\left\{v_{i}: i \in I\right\}$ for a Hilbert $C^{*}$-module satisfies $\langle x, x\rangle=\sum_{i \in I}\left\langle x, v_{i}\right\rangle\left\langle v_{i}, x\right\rangle$ for all $x \in V$, with the norm convergence ([2, Theorem 1]).

Recall that, if $A$ is a $C^{*}$-subalgebra of $\mathbf{K}(H)$ and $e \in A$ a non-zero projection, the condition $e A e=\mathbf{C} e$ is equivalent to the minimality of $e$ (i.e., the only subprojections of $e$ in $A$ are 0 and $e$ ) [1, Lema 1.4.1]. Minimal projections in $\mathbf{K}(H)$ are exactly orthogonal projections of rank 1 .

Clearly, an arbitrary Hilbert $C^{*}$-module does not possess an orthonormal basis, since there are $C^{*}$-algebras without projections. It is known that every Hilbert $C^{*}$-module $V$ over $\mathbf{K}(H)$ possesses an orthonormal basis; furthermore, for a fixed orthogonal projection $e \in \mathbf{K}(H)$ of rank 1 , there is an orthonormal basis $\{v: i: i \in$ $I\}$ for $V$ such that $\left\langle v_{i}, v_{i}\right\rangle=e$ for all $i \in I$.

In a Hilbert $\mathbf{K}(H)$-module, the condition of the minimality of supporting projections $e_{i}=\left\langle v_{i}, v_{i}\right\rangle, i \in I$, ensures that all orthonormal bases have the same cardinality ([2, Theorem 2]). For a countably generated Hilbert $\mathbf{K}(H)$-module, a set of indices for (all) orthonormal bases is countable. (By choosing an orthonormal basis 
$\left\{v_{i}: i \in I\right\}$ such that $\left\langle v_{i}, v_{i}\right\rangle=e, i \in I$, for some orthogonal projection $e \in \mathbf{K}(H)$ of rank 1, the last statement proves in the same way as in the Hilbert space case.) So, every orthonormal basis for a countably generated Hilbert $\mathbf{K}(H)$-module $V$ is a standard Parseval frame for $V$.

The paper is organized as follows.

In Section 2 we study standard frames for arbitrary countably generated Hilbert $C^{*}$-modules. We first show that an adjointable operator between Hilbert $C^{*}$ modules is bounded below with respect to the norm if and only if it is bounded below with respect to the inner product; furthermore, this is equivalent to the surjectivity of its adjoint operator. The first equivalence implies that, in the definition of standard frames, we can replace (1.1) with $C\|x\|^{2} \leq\left\|\sum_{i \in I}\left\langle x, f_{i}\right\rangle\left\langle f_{i}, x\right\rangle\right\| \leq D\|x\|^{2}$ for all $x \in V$ (Theorem 2.6). From the second equivalence we conclude that surjective adjointable operators preserve standard frames (Theorem 2.5).

In Section 3 we discuss standard frames $\left\{f_{i}: i \in I\right\}$ for which there exists a family of projections $\left\{e_{i}: i \in I\right\}$ such that $e_{i} A e_{i}=\mathbf{C} e_{i}$ and $f_{i}=f_{i} e_{i}$ for every $i \in I$. Surjective images of orthonormal bases are frames of this form. We prove that only a Hilbert $C^{*}$-module $V$ for which $\langle V, V\rangle$ is a $C C R$-algebra admits such frames. Discussion is mainly restricted to countably generated Hilbert $\mathbf{K}(H)$ modules, where such frames always exist; moreover, for every orthogonal projection $e \in \mathbf{K}(H)$ of rank 1, there is a frame $\left\{f_{i}: i \in I\right\}$ such that $f_{i}=f_{i} e$ for all $i \in I$. We show that frames $\left\{f_{i}: i \in I\right\}$ for a countably generated Hilbert $\mathbf{K}(H)$ module $V$ such that $\left\langle f_{i}, f_{i}\right\rangle=e, i \in I$, correspond to frames for a Hilbert space $V_{e}=\{v e: v \in V\}$ (Theorem 3.4).

\section{Some Properties of STANDARD MOdUlar Frames}

The results we obtain in this section are the consequences of the statement which generalizes the well known fact: a bounded linear operator between Hilbert spaces is surjective if and only if its adjoint is bounded below.

Proposition 2.1. Let $A$ be a $C^{*}$-algebra, $V$ and $W$ Hilbert $A$-modules, and $T \in$ $\mathbf{B}(V, W)$. The following statements are mutually equivalent:

(1) $T$ is surjective.

(2) $T^{*}$ is bounded below with respect to the norm, i.e., there is $m>0$ such that $\left\|T^{*} x\right\| \geq m\|x\|$ for all $x \in V$.

(3) $T^{*}$ is bounded below with respect to the inner product, i.e., there is $m^{\prime}>0$ such that $\left\langle T^{*} x, T^{*} x\right\rangle \geq m^{\prime}\langle x, x\rangle$ for all $x \in V$.

Proof. (1) $\Rightarrow(3)$ : Suppose $T$ is surjective. Then $\operatorname{Im} T=W$ is closed. It follows from [12, Theorem 3.2] that $\operatorname{Im} T^{*}$ is also closed, $\operatorname{Ker} T \oplus \operatorname{Im} T^{*}=V$ and $\operatorname{Ker} T^{*} \oplus \operatorname{Im} T=$ $W$. We shall prove that $T T^{*}$ is bijective.

If $T T^{*} x=0$ for some $x \in V$, then $T^{*} x \in \operatorname{Ker} T \cap \operatorname{Im} T^{*}=\{0\}$, hence $T^{*} x=0$. Now $x \in \operatorname{Ker} T^{*}=(\operatorname{Im} T)^{\perp}=W^{\perp}=\{0\}$ implies $x=0$. This proves that $T T^{*}$ is injective.

Let $z$ be an arbitrarily chosen element of $W$. $T$ is surjective, so $z=T y$ for some $y \in V$. There are $y_{1} \in \operatorname{Ker} T$ and $x \in W$ such that $y=y_{1} \oplus T^{*} x$. Then $z=T y=T\left(y_{1} \oplus T^{*} x\right)=T T^{*} x$; therefore $T T^{*}$ is surjective.

Since $T T^{*}$ is a positive invertible element of the $C^{*}$-algebra $\mathbf{B}(V)$, we have

$$
0 \leq\left(T T^{*}\right)^{-1} \leq\left\|\left(T T^{*}\right)^{-1}\right\| \mathrm{id}_{V} \Rightarrow T T^{*} \geq\left(\left\|\left(T T^{*}\right)^{-1}\right\|\right)^{-1} \mathrm{id}_{V}
$$


where id ${ }_{V}$ stands for the identity operator on $V$. Denoting $m^{\prime}=\left\|\left(T T^{*}\right)^{-1}\right\|^{-1}$ we get $T T^{*}-m^{\prime}$ id $_{V} \geq 0$. By [12, Lemma 4.1], this is equivalent to

$$
\left\langle\left(T T^{*}-m^{\prime} \operatorname{id}_{V}\right) x, x\right\rangle \geq 0
$$

for all $x \in V$, i.e., $\left\langle T^{*} x, T^{*} x\right\rangle \geq m^{\prime}\langle x, x\rangle$ for all $x \in V$.

The implication $(3) \Rightarrow(2)$ is trivial.

$(2) \Rightarrow(1)$ : Suppose that $T^{*}$ is bounded below with respect to the norm. Then $T^{*}$ is clearly injective, and it is easy to see that $\operatorname{Im} T^{*}$ is closed. Then $T$ has the closed range, again by [12, Theorem 3.2], and $W=\operatorname{Ker} T^{*} \oplus \operatorname{Im} T=\{0\} \oplus \operatorname{Im} T=\operatorname{Im} T$. Hence $T$ is surjective.

Corollary 2.2. Let $A$ be a $C^{*}$-algebra, $V$ a Hilbert $A$-module, and $T \in \mathbf{B}(V)$ such that $T=T^{*}$. The following statements are mutually equivalent:

(1) $T$ is surjective.

(2) There are $m, M>0$ such that $m\|x\| \leq\|T x\| \leq M\|x\|$ for all $x \in V$.

(3) There are $m^{\prime}, M^{\prime}>0$ such that $m^{\prime}\langle x, x\rangle \leq\langle T x, T x\rangle \leq M^{\prime}\langle x, x\rangle$ for all $x \in V$.

Remark 2.3. An operator $T \in \mathbf{B}(V)$ is said to be coercive if there is a positive constant $m$ such that $\left\langle T^{*} x, T^{*} x\right\rangle \geq m\langle x, x\rangle$ holds for all $x \in V$. It follows from Proposition 2.1 that coercive operators in $\mathbf{B}(V)$ are exactly surjections in $\mathbf{B}(V)$.

Theorem 2.4. Let $A$ be a $C^{*}$-algebra, $V$ a countably generated Hilbert A-module, $\left\{f_{i}: i \in I\right\}$ a sequence in $V$, and $\theta(x)=\left(\left\langle f_{i}, x\right\rangle\right)_{i \in I}$ for $x \in V$. The following statements are mutually equivalent:

(1) $\left\{f_{i}: i \in I\right\}$ is a standard frame for $V$.

(2) $\theta \in \mathbf{B}\left(V, \ell_{2}(A)\right)$ and $\theta$ is bounded below.

(3) $\theta \in \mathbf{B}\left(V, \ell_{2}(A)\right)$ and $\theta^{*}$ is surjective.

Proof. It follows from [8, Theorem 4.1] and Proposition 2.1 since

$$
\langle\theta x, \theta x\rangle=\sum_{i \in I}\left\langle x, f_{i}\right\rangle\left\langle f_{i}, x\right\rangle, \quad x \in V .
$$

Another direct consequence of Proposition 2.1 is that surjective adjointable operators preserve standard frames.

Theorem 2.5. Let $A$ be a $C^{*}$-algebra, $V$ and $W$ countably generated Hilbert $A$ modules, and $T \in \mathbf{B}(V, W)$ surjective. If $\left\{f_{i}: i \in I\right\}$ is a standard frame for $V$ with frame bounds $C$ and $D$, then $\left\{T f_{i}: i \in I\right\}$ is a standard frame for $W$ with frame bounds $\frac{C}{\left\|\left(T T^{*}\right)^{-1}\right\|}$ and $D\|T\|^{2}$.

Proof. Since $\left\{f_{i}: i \in I\right\}$ is a standard frame for $V$, and since $T^{*} y \in V$ for all $y \in W$, we have

$$
C\left\langle T^{*} y, T^{*} y\right\rangle \leq \sum_{i \in I}\left\langle T^{*} y, f_{i}\right\rangle\left\langle f_{i}, T^{*} y\right\rangle \leq D\left\langle T^{*} y, T^{*} y\right\rangle, \quad y \in W .
$$

From the proof of Proposition 2.1 we have $\left\langle T^{*} y, T^{*} y\right\rangle \geq\left\|\left(T T^{*}\right)^{-1}\right\|^{-1}\langle y, y\rangle$ for all $y \in W$, since $T$ is surjective. It follows that

$$
\frac{C}{\left\|\left(T T^{*}\right)^{-1}\right\|}\langle y, y\rangle \leq \sum_{i \in I}\left\langle y, T f_{i}\right\rangle\left\langle T f_{i}, y\right\rangle \leq D\|T\|^{2}\langle y, y\rangle, \quad y \in W .
$$


We conclude this section with the result which states that the condition (1.1) from the definition of standard frames can be replaced with a weaker one.

Theorem 2.6. Let $A$ be a $C^{*}$-algebra, $V$ a countably generated Hilbert $A$-module, and $\left\{f_{i}: i \in I\right\}$ a sequence in $V$ such that $\sum_{i \in I}\left\langle x, f_{i}\right\rangle\left\langle f_{i}, x\right\rangle$ converges in norm for every $x \in V$. Then $\left\{f_{i}: i \in I\right\}$ is a standard frame for $V$ if and only if there are constants $C, D>0$ such that

$$
C\|x\|^{2} \leq\left\|\sum_{i \in I}\left\langle x, f_{i}\right\rangle\left\langle f_{i}, x\right\rangle\right\| \leq D\|x\|^{2}, \quad x \in V .
$$

Proof. Evidently, every standard frame for $V$ satisfies (2.1).

For the converse we suppose that a sequence $\left\{f_{i}: i \in I\right\}$ fulfills (2.1). For an arbitrary $x \in V$ and a finite $J \subseteq I$ we define $x_{J}=\sum_{i \in J} f_{i}\left\langle f_{i}, x\right\rangle$. Then

$$
\begin{gathered}
\left\|x_{J}\right\|^{4}=\left\|\left\langle x_{J}, x_{J}\right\rangle\right\|^{2}=\left\|\left\langle x_{J}, \sum_{i \in J} f_{i}\left\langle f_{i}, x\right\rangle\right\rangle\right\|^{2}=\left\|\sum_{i \in J}\left\langle x_{J}, f_{i}\right\rangle\left\langle f_{i}, x\right\rangle\right\|^{2} \\
\leq\left\|\sum_{i \in J}\left\langle x_{J}, f_{i}\right\rangle\left\langle f_{i}, x_{J}\right\rangle\right\| \cdot\left\|\sum_{i \in J}\left\langle x, f_{i}\right\rangle\left\langle f_{i}, x\right\rangle\right\| \leq D\left\|x_{J}\right\|^{2}\left\|\sum_{i \in J}\left\langle x, f_{i}\right\rangle\left\langle f_{i}, x\right\rangle\right\|,
\end{gathered}
$$

therefore

$$
\left\|\sum_{i \in J} f_{i}\left\langle f_{i}, x\right\rangle\right\|^{2}=\left\|x_{J}\right\|^{2} \leq D\left\|\sum_{i \in J}\left\langle x, f_{i}\right\rangle\left\langle f_{i}, x\right\rangle\right\| .
$$

Since $J$ is arbitrary, the series $\sum_{i \in I} f_{i}\left\langle f_{i}, x\right\rangle$ converges and

$$
\left\|\sum_{i \in I} f_{i}\left\langle f_{i}, x\right\rangle\right\|^{2} \leq D\left\|\sum_{i \in I}\left\langle x, f_{i}\right\rangle\left\langle f_{i}, x\right\rangle\right\| \leq D^{2}\|x\|^{2} \Rightarrow\left\|\sum_{i \in I} f_{i}\left\langle f_{i}, x\right\rangle\right\| \leq D\|x\| .
$$

Since $x \in V$ is arbitrarily chosen, the operator

$$
T: V \rightarrow V, \quad x \mapsto \sum_{i \in I} f_{i}\left\langle f_{i}, x\right\rangle
$$

is well defined, bounded and $A$-linear. It is easy to check that $\langle T x, y\rangle=\langle x, T y\rangle$ for all $x, y \in V$, so $T \in \mathbf{B}(V)$ and $T=T^{*}$. From $\langle T x, x\rangle=\sum_{i \in I}\left\langle x, f_{i}\right\rangle\left\langle f_{i}, x\right\rangle \geq 0$ for all $x \in V$, it follows that $T \geq 0$. Now (2.1) and $\left\langle T^{1 / 2} x, T^{1 / 2} x\right\rangle=\sum_{i \in I}\left\langle x, f_{i}\right\rangle\left\langle f_{i}, x\right\rangle$ imply $\sqrt{C}\|x\| \leq\left\|T^{1 / 2} x\right\| \leq \sqrt{D}\|x\|$ for all $x \in V$. By Corollary 2.2, there are constants $C^{\prime}, D^{\prime}>0$ such that

$$
C^{\prime}\langle x, x\rangle \leq\left\langle T^{1 / 2} x, T^{1 / 2} x\right\rangle=\sum_{i \in I}\left\langle x, f_{i}\right\rangle\left\langle f_{i}, x\right\rangle \leq D^{\prime}\langle x, x\rangle, \quad x \in V .
$$

This proves that $\left\{f_{i}: i \in I\right\}$ is a standard frame for $V$.

\section{On a Class of Frames fOr Hilbert $\mathbf{K}(H)$-MOdules}

The existence of standard frames in countably generated Hilbert $\mathbf{K}(H)$-modules $V$ follows from the existence of orthonormal bases. If $T \in \mathbf{B}(V)$ is a surjection and $\left\{v_{i}: i \in I\right\}$ an orthonormal basis for $V$, then $\left\{T v_{i}: i \in I\right\}$ is a standard frame for $V$ which satisfies $T v_{i}=T\left(v_{i} e_{i}\right)=\left(T v_{i}\right) e_{i}$, where $e_{i}:=\left\langle v_{i}, v_{i}\right\rangle$ is an orthogonal projection of rank 1 for every $i \in I$. However, not every standard frame in a Hilbert $\mathbf{K}(H)$-module is of this type, as we show in the next example. 
Example 3.1. Let $\left\{v_{i}: i \in I\right\}$ be an orthonormal basis for a countably and not finitely generated Hilbert $\mathbf{K}(H)$-module $V$ with property $e_{i} e_{j}=0, i \neq j$, where $e_{i}=\left\langle v_{i}, v_{i}\right\rangle$. (Such a basis can always be constructed by following the procedure described in 2, Remark 4(d)].) Let $I=\bigcup_{j=1}^{\infty} I_{j}$ be a partition of $I$ such that $\left|I_{j}\right|=j$. Let $f_{j}=\sum_{i \in I_{j}} v_{i} \in V$. Since $\left\langle x, v_{j}\right\rangle\left\langle v_{i}, x\right\rangle=\left\langle x, v_{j} e_{j}\right\rangle\left\langle v_{i} e_{i}, x\right\rangle=\left\langle x, v_{j}\right\rangle e_{j} e_{i}\left\langle v_{i}, x\right\rangle=$ $\delta_{i j}\left\langle x, v_{j}\right\rangle\left\langle v_{i}, x\right\rangle$ for all $x \in V$, we have

$$
\left\langle x, f_{j}\right\rangle\left\langle f_{j}, x\right\rangle=\left\langle x, \sum_{i \in I_{j}} v_{i}\right\rangle\left\langle\sum_{i \in I_{j}} v_{i}, x\right\rangle=\sum_{i, j \in I_{j}}\left\langle x, v_{j}\right\rangle\left\langle v_{i}, x\right\rangle=\sum_{i \in I_{j}}\left\langle x, v_{i}\right\rangle\left\langle v_{i}, x\right\rangle
$$

and then

$$
\langle x, x\rangle=\sum_{i \in I}\left\langle x, v_{i}\right\rangle\left\langle v_{i}, x\right\rangle=\sum_{j \in J}\left\langle x, f_{j}\right\rangle\left\langle f_{j}, x\right\rangle .
$$

This means that $\left\{f_{j}: j \in J\right\}$ is a standard Parseval frame for $V$ such that $\left\langle f_{j}, f_{j}\right\rangle=$ $\sum_{i \in I_{j}} e_{i}$ is a projection with $\operatorname{dim} \operatorname{Im}\left\langle f_{i}, f_{i}\right\rangle=\left|I_{j}\right|=j$ for all $j \in J$.

Proposition 3.2. Let $V$ be a countably and not finitely generated Hilbert $\mathbf{K}(H)$ module. Let $\left\{f_{i}: i \in I\right\}$ be a standard frame for $V$ such that $f_{i}=f_{i} e_{i}$ for some orthogonal projections $e_{i}, i \in I$, of rank 1 . Then there is an orthonormal basis $\left\{v_{i}: i \in I\right\}$ and a surjection $T \in \mathbf{B}(V)$ such that $T v_{i}=f_{i}, i \in I$.

Proof. Let $C$ and $D$ be frame bounds. Let $\left\{v_{i}: i \in I\right\}$ be an orthonormal basis such that $v_{i}=v_{i} e_{i}, i \in I$. (We may assume that the sets of indices for a standard frame and a basis are the same, since they are both infinite subsets of $\mathbf{N}$.)

We first show that for every $x \in V$ the series $\sum_{i \in I} f_{i}\left\langle v_{i}, x\right\rangle$ converges. Let $J$ be a finite subset of $I$ and $x_{J}=\sum_{i \in J} f_{i}\left\langle v_{i}, x\right\rangle$. Then

$$
\begin{gathered}
\left\|x_{J}\right\|^{4}=\left\|\left\langle x_{J}, x_{J}\right\rangle\right\|^{2}=\left\|\left\langle\sum_{i \in J} f_{i}\left\langle v_{i}, x\right\rangle, x_{J}\right\rangle\right\|^{2}=\left\|\sum_{i \in J}\left\langle x, v_{i}\right\rangle\left\langle f_{i}, x_{J}\right\rangle\right\|^{2} \\
\leq\left\|\sum_{i \in J}\left\langle x, v_{i}\right\rangle\left\langle v_{i}, x\right\rangle\right\|\left\|\sum_{i \in J}\left\langle x_{J}, f_{i}\right\rangle\left\langle f_{i}, x_{J}\right\rangle\right\| \leq\left\|\sum_{i \in J}\left\langle x, v_{i}\right\rangle\left\langle v_{i}, x\right\rangle\right\| \cdot D\left\|x_{J}\right\|^{2},
\end{gathered}
$$

from where we get $\left\|x_{J}\right\|^{2} \leq D\left\|\sum_{i \in J}\left\langle x, v_{i}\right\rangle\left\langle v_{i}, x\right\rangle\right\|$, that is,

$$
\left\|\sum_{i \in J} f_{i}\left\langle v_{i}, x\right\rangle\right\|^{2} \leq D\left\|\sum_{i \in J}\left\langle x, v_{i}\right\rangle\left\langle v_{i}, x\right\rangle\right\|, \text { for every finite } J \subseteq I .
$$

Since $\sum_{i \in I}\left\langle x, v_{i}\right\rangle\left\langle v_{i}, x\right\rangle$ converges in norm ([2, Theorem 1]), it follows that the series $\sum_{i \in I} f_{i}\left\langle v_{i}, x\right\rangle$ converges.

Similarly, we check that the series $\sum_{i \in I} v_{i}\left\langle f_{i}, x\right\rangle$ converges for every $x \in V$.

Now we can define the operators $T, R: V \rightarrow V$ by $T x=\sum_{i \in I} f_{i}\left\langle v_{i}, x\right\rangle$ and $R x=\sum_{i \in I} v_{i}\left\langle f_{i}, x\right\rangle$. It is straightforward to see that $\langle T x, y\rangle=\langle x, R y\rangle$ for all $x, y \in V$. Therefore $T \in \mathbf{B}(V)$ and $R=T^{*}$. From Proposition 2.1] and

$$
C\langle x, x\rangle \leq \sum_{i \in I}\left\langle x, f_{i}\right\rangle\left\langle f_{i}, x\right\rangle=\left\langle T^{*} x, T^{*} x\right\rangle, \quad x \in V,
$$

it follows that $T$ is surjective.

It only remains to note that $T v_{i}=f_{i}\left\langle v_{i}, v_{i}\right\rangle=f_{i} e_{i}=f_{i}$ for all $i \in I$.

A Hilbert $\mathbf{K}(H)$-module contains a Hilbert space $V_{e}$ with respect to the inner product $(x, y)=\operatorname{tr}(\langle y, x\rangle)$, where 'tr' means the trace. More precisely, for a fixed orthogonal projection $e \in \mathbf{K}(H)$ of rank $1, V_{e}$ is given as the set of all $x e, x \in V$. 
Also, for all $x, y \in V_{e}$ we obtain that $\langle x, y\rangle=(y, x) e . V_{e}$ is an invariant subspace for each $T$ in $\mathbf{B}(V)$ and the map

$$
T \mapsto T \mid V_{e}, \quad \mathbf{B}(V) \rightarrow \mathbf{B}\left(V_{e}\right)
$$

establishes an isomorphism of $C^{*}$-algebras, where $\mathbf{B}\left(V_{e}\right)$ denotes the $C^{*}$-algebra of all bounded operators on $V_{e}$. It is known that a family $\left\{v_{i}: i \in I\right\} \subseteq V_{e}$ is an orthonormal basis for $V$ if and only if it is an orthonormal basis for $V_{e}$. (The proofs can be found in [2, Remark 4, Theorem 5]).) We extend the last statement to a standard frame $\left\{f_{i}: i \in I\right\}$ contained in $V_{e}$. First we need a lemma which describes some properties of the isomorphism (3.1).

Lemma 3.3. Let $V$ be a Hilbert $\mathbf{K}(H)$-module, $e \in \mathbf{K}(H)$ an orthogonal projection of rank 1 , and $T \in \mathbf{B}(V)$. The following statements hold:

(1) $T$ is bounded below if and only if $T \mid V_{e} \in \mathbf{B}\left(V_{e}\right)$ is bounded below.

(2) $T$ is surjective if and only if $T \mid V_{e} \in \mathbf{B}\left(V_{e}\right)$ is surjective.

Proof. (1) First observe that if $T \mid V_{e} \in \mathbf{B}\left(V_{e}\right)$ is a positive operator on the Hilbert space $V_{e}$, then $T \in \mathbf{B}(V)$ is a positive element of the $C^{*}$-algebra $\mathbf{B}(V)$. This is a consequence of the fact that the map $T \mapsto T \mid V_{e}$ is an isomorphism of $C^{*}$-algebras.

Suppose $T_{e}:=T \mid V_{e}$ is bounded below. Let $m>0$ be such that $\left\|T_{e}(x e)\right\| \geq$ $m\|x e\|$ for all $x \in V$. In other words, $T_{e}^{*} T_{e}-m^{2} \mathrm{id}_{V_{e}}$ is a positive operator on the Hilbert space $V_{e}$. By the observation from the beginning of the proof, we get $T^{*} T-m^{2} \operatorname{id}_{V} \geq 0$, i.e., $\left\langle\left(T^{*} T-m^{2} \operatorname{id}_{V}\right) x, x\right\rangle \geq 0$ for all $x \in V$. Now we have $\langle T x, T x\rangle \geq m^{2}\langle x, x\rangle$, and then $\|T x\| \geq m\|x\|$ for all $x \in V$.

The opposite statement is obvious.

(2) It follows from (1) and Proposition 2.1

Theorem 3.4. Let $V$ be a countably generated Hilbert $\mathbf{K}(H)$-module, $e \in \mathbf{K}(H)$ an orthogonal projection of rank 1 , and $\left\{f_{i}: i \in I\right\}$ a sequence of vectors in $V_{e}$. Then $\left\{f_{i}: i \in I\right\}$ is a standard frame for the Hilbert $\mathbf{K}(H)$-module $V$ with frame bounds $C$ and $D$ if and only if $\left\{f_{i}: i \in I\right\}$ is a frame for the Hilbert space $V_{e}$ with frame bounds $C$ and $D$.

Proof. Suppose that $\left\{f_{i}: i \in I\right\}$ is a standard frame for a Hilbert $\mathbf{K}(H)$-module $V$ with frame bounds $C$ and $D$. It means that

$$
C\langle x, x\rangle \leq \sum_{i \in I}\left\langle x, f_{i}\right\rangle\left\langle f_{i}, x\right\rangle \leq D\langle x, x\rangle, \quad x \in V .
$$

Since $\langle x e, y e\rangle=(y e, x e) e$ for all $x e, y e \in V_{e}$, by choosing $x e$ instead of $x$ in the above inequalities, we get

$$
C(x e, x e) e \leq \sum_{i \in I}\left(x e, f_{i}\right)\left(f_{i}, x e\right) e \leq D(x e, x e) e, \quad x \in V,
$$

which implies $C(x, x) \leq \sum_{i \in I}\left(x, f_{i}\right)\left(f_{i}, x\right) \leq D(x, x)$ for all $x \in V_{e}$. It proves that $\left\{f_{i}: i \in I\right\}$ is a frame for the Hilbert space $V_{e}$ with frame bounds $C$ and $D$.

Now suppose that $\left\{f_{i}: i \in I\right\} \subseteq V_{e}$ is a frame for the Hilbert space $V_{e}$ with frame bounds $C$ and $D$.

First we assume that $V$ is finitely generated. Let $\left\{v_{1}, \ldots, v_{n}\right\} \subseteq V_{e}$ be an orthonormal basis for $V$ and $S_{e} \in \mathbf{B}\left(V_{e}\right)$ the frame operator associated to the (Hilbert 
space) frame $\left\{f_{i}: i \in I\right\}$. Then

$$
x e=\sum_{i \in I} S_{e}^{\frac{1}{2}} f_{i}\left(x e, S_{e}^{\frac{1}{2}} f_{i}\right)=\sum_{i \in I} S_{e}^{\frac{1}{2}} f_{i}\left\langle S_{e}^{\frac{1}{2}} f_{i}, x e\right\rangle, \quad x e \in V_{e} .
$$

Since $x=\sum_{j=1}^{n} v_{i}\left\langle v_{i}, x\right\rangle$ for all $x \in V$, and since $v_{1}, \ldots, v_{n} \in V_{e}$, we immediately get that for all $x \in V, x=\sum_{i \in I} S_{e}^{\frac{1}{2}} f_{i}\left\langle S_{e}^{\frac{1}{2}} f_{i}, x\right\rangle$ holds. This proves that $\left\{S_{e}^{\frac{1}{2}} f_{i}: i \in I\right\}$ is a Parseval standard frame for $V$. Let $S \in \mathbf{B}(V)$ be the unique extension of $S_{e} \in \mathbf{B}\left(V_{e}\right)$. Since $S_{e}$ is invertible and positive, $S \in \mathbf{B}(V)$ is also invertible and positive. Therefore $S^{-\frac{1}{2}}$ preserves standard frames, so the sequence $\left\{S^{-\frac{1}{2}}\left(S_{e}^{\frac{1}{2}} f_{i}\right)\right.$ : $i \in I\}=\left\{S_{e}^{-\frac{1}{2}} S_{e}^{\frac{1}{2}} f_{i}: i \in I\right\}=\left\{f_{i}: i \in I\right\}$ is a standard frame for $V$.

Now we assume that $V$ is not finitely generated. Let $\left\{v_{i}: i \in I\right\}$ be an orthonormal basis for $V$ such that $v_{i}=v_{i} e$ for all $i \in I$. Then $\left\{v_{i}: i \in I\right\}$ is an orthonormal basis for the Hilbert space $V_{e}$. Let $T_{e}: V_{e} \rightarrow V_{e}$ be the operator defined as $T_{e}(x e)=\sum_{i \in I} f_{i}\left\langle v_{i}, x e\right\rangle$. As in the proof of Proposition 3.2 we show that $T_{e}$ is well defined, $T_{e} \in \mathbf{B}\left(V_{e}\right)$ and $T_{e}$ is surjective. Let $T \in \mathbf{B}(V)$ be the unique extension of $T_{e} \in \mathbf{B}\left(V_{e}\right)$. By the previous lemma, $T$ is surjective. Now Theorem 2.5 implies that $\left\{f_{i}: i \in I\right\}$ is a standard frame for $V$, since $T\left(v_{i}\right)=T_{e}\left(v_{i}\right)=f_{i}$ for all $i \in I$.

The concept of an orthonormal basis has been introduced in Hilbert $C^{*}$-modules over an arbitrary $C^{*}$-algebra. Obviously, there are Hilbert $C^{*}$-modules which do not possess an orthonormal basis. Actually, if a Hilbert $C^{*}$-module $V$ possesses an orthonormal basis, then $\langle V, V\rangle$ has to be a $C C R$-algebra. We prove this in the next theorem.

Theorem 3.5. Let $A$ be a $C^{*}$-algebra and $V$ a full countably generated Hilbert $A$ module. Let $\left\{e_{i}: i \in I\right\}$ be a family of projections in $A$ such that $e_{i} A e_{i}=\mathbf{C} e_{i}, i \in I$, and $\left\{f_{i}: i \in I\right\}$ a standard frame for $V$ such that $f_{i}=f_{i} e_{i}, i \in I$. Then $A$ is a $C C R$-algebra. In particular, if $V$ possesses an orthonormal basis, then $A$ is a $C C R$-algebra.

Proof. By the definition of a $C C R$-algebra we need to show that for every irreducible representation $\varphi: A \rightarrow \mathbf{B}(H), \varphi(A) \subseteq \mathbf{K}(H)$ holds.

Let $0 \neq \varphi: A \rightarrow \mathbf{B}(H)$ be an irreducible representation of $A$. Then $e_{i} A e_{i}=\mathbf{C} e_{i}$ implies $\varphi\left(e_{i}\right) \varphi(A) \varphi\left(e_{i}\right)=\mathbf{C} \varphi\left(e_{i}\right)$ for every $i \in I$.

Let $i \in I$ be such that $\varphi\left(e_{i}\right) \neq 0$. Now $\varphi\left(e_{i}\right)$ is a non-zero projection, so there is a non-zero vector $\xi_{0} \in H$ which belongs to $\operatorname{Im} \varphi\left(e_{i}\right)$. Then $\varphi\left(e_{i}\right) \xi_{0}=\xi_{0}$ and

$$
\varphi\left(e_{i}\right) \varphi(A) \varphi\left(e_{i}\right) \xi_{0}=\mathbf{C} \varphi\left(e_{i}\right) \xi_{0} \Rightarrow \varphi\left(e_{i}\right) \varphi(A) \xi_{0}=\mathbf{C} \xi_{0} .
$$

$\varphi$ is irreducible, therefore it is a cyclic representation of $A$, and every non-zero vector is cyclic for $\varphi$. In particular, $\xi_{0}$ is a cyclic vector for $\varphi$. Therefore

$$
\{0\} \neq \varphi\left(e_{i}\right) H=\varphi\left(e_{i}\right) \overline{\varphi(A) \xi_{0}} \subseteq \overline{\varphi\left(e_{i}\right) \varphi(A) \xi_{0}}=\mathbf{C} \xi_{0} \Rightarrow \operatorname{Im} \varphi\left(e_{i}\right)=\mathbf{C} \xi_{0} .
$$

This proves that $\varphi\left(e_{i}\right) \in \mathbf{K}(H)$ for every $i \in I$.

Let $S \in \mathbf{B}(V)$ be the frame operator associated to $\left\{f_{i}: i \in I\right\}$. From the reconstruction formula (1.2) we have $\langle x, y\rangle=\sum_{i \in I}\left\langle x, S f_{i}\right\rangle\left\langle f_{i}, y\right\rangle$ and then

$$
\varphi(\langle x, y\rangle)=\sum_{i \in I} \varphi\left(\left\langle x, S f_{i}\right\rangle\right) \varphi\left(\left\langle f_{i}, y\right\rangle\right), \quad x, y \in V .
$$


From $f_{i}=f_{i} e_{i}$ and compactness of $\varphi\left(e_{i}\right)$ it follows that

$$
\varphi\left(\left\langle x, S f_{i}\right\rangle\right) \varphi\left(\left\langle f_{i}, y\right\rangle\right)=\varphi\left(\left\langle x, S f_{i}\right\rangle\right) \varphi\left(e_{i}\right) \varphi\left(\left\langle f_{i}, y\right\rangle\right) \in \mathbf{K}(H), \quad x, y \in V, i \in I .
$$

Finally, we get $\varphi(\langle x, y\rangle) \in \mathbf{K}(H)$ for all $x, y \in V$, as the convergence in (3.2) is in norm. Since $V$ is full, we conclude that $\varphi(A) \subseteq \mathbf{K}(H)$.

This finishes our proof.

The converse of the previous theorem does not hold. For example, we can take an arbitrary Hilbert $C^{*}$-module over the $C^{*}$-algebra $A=C([0,1])$ of all continuous complex functions on the unit segment $[0,1] . A$ is a $C C R$-algebra, since it is commutative, and the only projection $e \in A$ which satisfies $e A e=\mathbf{C} e$ is the constant function 0 .

Remark 3.6. Frames of subspaces for a separable Hilbert space have been recently introduced and studied in [4. We can generalize their definition for Hilbert $\mathbf{K}(H)$ modules in the following way.

Let $V$ be a countably generated Hilbert $\mathbf{K}(H)$-module, $\left\{W_{i}: i \in I\right\}(I \subseteq \mathbf{N})$ a family of closed submodules of $V$, and $\left\{\lambda_{i}: i \in I\right\}$ a family of weights, i.e., a family of positive numbers. We say that $\left\{W_{i}: i \in I\right\}$ is a standard frame of submodules for $V$ with respect to a family of weights $\left\{\lambda_{i}: i \in I\right\}$, if there are constants $C, D>0$ such that

$$
C\langle x, x\rangle \leq \sum_{i \in I} \lambda_{i}^{2}\left\langle\pi_{i}(x), \pi_{i}(x)\right\rangle \leq D\langle x, x\rangle, \quad x \in V,
$$

where $\pi_{i} \in \mathbf{B}(V)$ denotes the orthogonal projection on $W_{i}$ for every $i \in I$, and convergence of the sum in the middle of (3.3) is in norm.

Let us fix an orthogonal projection $e \in \mathbf{K}(H)$ of rank 1. It can be proved that a family of closed submodules $\left\{W_{i}: i \in I\right\}$ is a standard frame of submodules for $V$ with respect to the family of weights $\left\{\lambda_{i}: i \in I\right\}$ if and only if $\left\{\left(W_{i}\right)_{e}: i \in I\right\}$ is a frame of subspaces for $V_{e}$ with respect to the family of weights $\left\{\lambda_{i}: i \in I\right\}$. Therefore many statements from [4] can be extended to countably generated Hilbert $\mathbf{K}(H)$-modules. This will be done in our subsequent paper.

\section{ACKNowledgement}

The author would like to thank Professors Damir Bakić and Boris Guljaš for helpful discussions. Thanks are also due to the referee for useful comments.

\section{REFERENCES}

[1] W. B. Arveson, An invitation to $C^{*}$-algebras, Graduate Texts in Mathematics, No. 39. Springer-Verlag, New York-Heidelberg, 1976. MR0512360 (58:23621)

[2] D. Bakić, B. Guljaš, Hilbert $C^{*}$-modules over $C^{*}$-algebras of compact operators, Acta Sci. Math. (Szeged) 68 (2002), no. 1-2, 249-269. MR1916579 (2003f:46092)

[3] P. G. Casazza, The art of frame theory, Taiwanese J. Math 4 (2000), 129-201. MR1757401 (2001f:42046)

[4] P. G. Casazza, G. Kutyniok, Frames of subspaces, wavelets, frames and operator theory, 87-113, Contemp. Math., 345, Amer. Math. Soc., Providence, RI, 2004. MR2066823 (2005e:42090)

[5] J. Dixmier, C*-algebras, North-Holland, Amsterdam, 1977. MR.0458185 (56:16388)

[6] R. J. Duffin, A. C. Schaeffer, A class of nonharmonic Fourier series, Trans. Amer. Math. Soc. 72 (1952). 341-366. MR0047179(13:839a)

[7] M. Frank, D. R. Larson, A module frame concept for Hilbert $C^{*}$-modules, 207-233, Contemp. Math., 247, Amer. Math. Soc., Providence, RI, 1999. MR.1738091(2001b:46094) 
[8] 2, 273-314. MR1938798 (2003i:42040)

[9] M. Frank, V. I. Paulsen, T. R. Tiballi, Symmetric approximation of frames and bases in Hilbert spaces, Trans. Amer. Math. Soc. 354 (2002), no. 2, 777-793. MR1862567 $(2002 \mathrm{j}: 42042)$

[10] A. Khosravi, N. A. Moslemipour, Basic properties of standard frame in Hilbert $C^{*}$-modules, Int. J. Appl. Math. 14 (2003), no. 3, 243-258. MR2067907 (2005b:46131)

[11] _ Frame operator and alternate dual modular frame, Int. J. Appl. Math. 13 (2003), no. 2, 177-189. MR2022092 (2005b:46154)

[12] C. Lance, Hilbert $C^{*}$-modules - a toolkit for operator algebraists, London Math. Soc. Lecture Note Series 210, Cambridge University Press, Cambridge, 1995. MR1325694 (96k:46100)

[13] I. Raeburn, S. J. Thompson, Countably generated Hilbert modules, the Kasparov stabilisation theorem, and frames in Hilbert modules, Proc. Amer. Math. Soc. 131 (2003), no. 5, 15571564. MR1949886 (2003j:46089)

Department of Mathematics, University of Zagreb, BijeničKa C. 30, 10000 Zagreb, Croatia

E-mail address: ljsekul@math.hr 